Das UN-Umweltprogramm UNEP wäre der natürliche Kandidat, die Arbeit verschiedener Netzwerke zu koordinieren und, soweit möglich, zu managen. UNEP könnte die multisektorale Arbeit durch die Identifikation von Problemfeldern, durch das Zusammenbringen verschiedener Akteure, durch Wissensmanagement und -verbreitung fördern. Um jedoch eine sinnvolle und erfolgreiche Arbeit zu ermöglichen, müsste zunächst eine Strategie entwickelt werden, die einem weiteren ziellosen Aktionismus vorbeugt. Außerdem sollten die verschiedenen internationalen Organisationen nach ihren komparativen Vorteilen eingesetzt werden. Die noch immer vorherrschende Konkurrenz und Verzettelung, die dazu führt, dass personelle und finanzielle Ressourcen unnötig gebunden und verschwendet werden, muss beendet werden und einer fruchtbaren Zusammenarbeit weichen. Zweifellos werden die institutionellen und politischen Widerstände groß sein, doch ohne Vision können die strukturellen Hürden des momentanen Systems der umweltpolitischen Zusammenarbeit nicht überwunden werden.

Auch in diesen Sinne mag die GEF als Vorbild dienen. Sie repräsentiert zwar noch lange nicht den Idealtypus einer netzwerkorientierten $\mathrm{Zu}$ sammenarbeit, hierzu sind unter anderem die institutionellen und politischen Widerstände zu stark. Wie gezeigt stellt sie gleichwohl einen Schritt in die richtige Richtung dar.

\section{Anmerkungen}

(1) Dieser Artikel basiert unter anderem auf der Zusammenarbeit mit Wolfgang Reinicke und seinem Team im Global Public Policy Network Project

(www.globalpublicpolicy.net).

(2) Vgl. zum Folgenden: Reinicke, Wolfgang: Global Public Policy. Governing without Government?, Washington DC 1998 sowie Reinicke, Wolfgang/ Deng, Francis: Critical Choices. IDRC, Ottawa 2000

(3) Vgl. Benner, Thorsten/ Witte, Jan Martin: Brücken im globalen System: Wandel und Zukunft internationaler Organisationen. In: Internationale Politik (im Erscheinen).

(4) Vgl. Streck, Charlotte: The Global Environment Facility - a Role Model for International Governance? In: Global Environmental Politics, Vol. 1, №. 2.

\section{Die Autorin}

Charlotte Streck ist Umweltjuristin bei der Weltbank. Kontakt: The World Bank, MC 6-446; 1818, H-Street, N.W.; Washington, D.C., 20433; USA. Tel. 001-202-4737331, Fax 5221573, E-mail: CStreck@worldbank.org

Internationale Unternehmerorganisationen und die Produktion globaler öffentlicher Güter

\title{
Übersehener Beitrag
}

\author{
Öffentliche Aufgaben werden zunehmend auf private Träger verschoben. Eine \\ solche Privatisierung öffentlicher Politik kann auch im internationalen Rahmen \\ beobachtet werden. Auch wenn Unternehmen als private Governance-Akteure \\ eigene Interessen verfolgen, können sie unter bestimmten Umständen auch \\ einen Beitrag zur Bereitstellung öffentlicher Güter wie Umwelt- und Gesund- \\ heitsschutz leisten. Dies wird am Beispiel Gefahrenminderung bei Farbstoffen \\ illustriert.
}

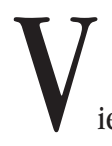
iele globale Regelungs- und Kontrollprobleme sind selbst durch koordiniertes zwischenstaatliches Handeln über internationale Organisationen nicht mehr in den Griff zu bekommen. Der einfache Grund ist, dass es staatlichen Akteuren im Prozess der gesellschaftlichen Differenzierung immer schwerer fällt, die hierfür nötigen Spezialressourcen zu mobilisieren und kollektiven Koordinationsleistungen zu erbringen. Selbst auf nationalstaatlicher Ebene sind staatliche Organisationen in der Formulierung und Implementation öffentlicher Politiken immer mehr auf die Kooperation von privaten Akteuren angewiesen. Derartige Beobachtungen wurden in den vergangenen Jahren unter dem Stichwort Politiknetzwerke diskutiert (1). Eine noch radikalere Antwort auf dieses Problem ist eine Form der Privatisierung öffentlicher Politik, bei der öffentliche Aufgaben insgesamt auf private Träger verschoben werden (2). Solche Prozesse öffentlichen Aufgabentransfers konnten in den vergangenen Jahren auch im internationalen Rahmen beobachtet werden, in dem öffentliche Aufgaben, die bislang von intergouvernementalen Organisationen wahrgenommen wurden, häufig an Nichtregierungsorganisationen (NGOs) delegiert werden.

\section{Neve Governance-Mechanismen}

In den Sozialwissenschaften werden diese institutionellen Arrangements, in denen sowohl öffentliche als auch private Akteure an der Regulierung globaler Entwicklungen mitwirken, unter dem Stichwort Global Governance diskutiert (3). Es wird erkannt, dass viele gesellschaftliche Steuerungsleistungen Abstimmungs- formen jenseits von Markt und Staat erfordern und auf Netzwerke oder andere alternative Governance-Mechanismen verweisen. Insbesondere in Situationen, in denen Marktkontrolle wie staatliche Steuerung unmöglich oder unerwünscht ist, ist globale institutionelle Steuerung auch durch nichtstaatliche Organisationen denkbar. Hierzu werden Organisationen und interorganisatorische Netzwerke im Menschenrechts- und Umweltbereich meist als Beispiele angeführt.

Private Steuerungsbeiträge beschränken sich jedoch nicht auf Organisationen, die unmittelbar an Gemeinwohlzielen orientiert sind, sondern können auch von Privatorganisationen erwartet werden, deren Hauptzweck eher in der Verfolgung von Partikularinteressen liegt. Voraussetzung ist eine Anreizkonstellation, die letztere mit Gemeinwohlinteressen in einer spezifischen Weise verknüpft. Aus einer solchen Perspektive ist es wichtig zu untersuchen, unter welchen Umständen private Organisationen solche Kapazitäten entwickeln können (Ressourcenproblematik), und unter welchen Bedingungen diese hierzu überhaupt das Interesse und den Willen aufbringen (Motivationsstruktur).

In den internationalen Beziehungen wird die Bereitstellung sogenannter öffentlicher Güter durch interstaatliches kollektives Handeln seit Jahrzehnten als interessante theoretische Problematik gesehen (4). Die strukturellen Eigenschaften öffentlicher Güter, Nichtausschlussfähigkeit und Nichtrivalität, implizieren bei einer individualistischen Verhaltenslogik, dass Individuen oder korporative Akteure nur wenig geneigt sind, solche Güter bereitzustellen. Daraus wird meist die Notwendigkeit abgeleitet, für solche Aufgaben öffentliche Organisationen zu errichten, die mit spezi- 
fischen Ressourcen und institutionellen Rahmenbedingungen auszustatten sind, um die privatautonomen Rationalitätsschranken und die damit verbundenen Kollektivgutprobleme zu überwinden.

\section{- Private Bereitstellung globaler öffentlicher Güter}

Die Beziehung zwischen öffentlichen Gütern und spezifischen Organisationsformen ist jedoch komplexer. Viele Beispiele belegen, dass öffentliche Güter unter bestimmten Bedingungen auch durch den Privatsektor erzeugt werden können. Ein besonderer Typus gemeinsam produzierter Güter, der auch unter rein privaten Arrangements relativ unproblematisch ist, umfasst die sogenannten Klubgüter, die im Zugang auf eine bestimmte Gruppe beschränkt werden können (5). Doch auch hiermit können - intendiert oder nicht intendiert - positive Externalitäten für andere verbunden sein.

Die grundlegende theoretische Kollektivgutproblematik gilt auch im Kontext globaler Politik. Kollektive Handlungsergebnisse einer privaten Bereitstellung können insofern gemischt sein, als sie sowohl Mitgliederinteressen befriedigen als auch zur Realisierung allgemeiner gesellschaftlicher Interessen beitragen.

Neben der Motivation ist eine wichtige Frage die Kompetenz: Interessanterweise sind die Gruppen, die in der Literatur der internationalen Beziehungen das größte Interesse gefunden haben, zwar in ihren Zielen am deutlichsten auf globale Kollektivgüter ausgerichtet, beispielsweise Umwelt- und Menschenrechtsverbände, jedoch in Bezug auf Ressourcen eher unterausgestattet. Dass auch organisierte Wirtschaftsinteressen sich auf globaler Ebene zusammenschließen und jenseits der Firmen- und Verbandsebene öffentliche Verantwortung übernehmen, hat bislang nur wenig Aufmerksamkeit erweckt (6). Gerade die Privatwirtschaft ist international durch eine Vielzahl von Verbänden vertreten und durch ihre organisationellen Ressourcen häufig besonders dafür geeignet, internationale Regulierungsfunktionen zu über-nehmen. 9

In der vergleichenden Sozial- und Politikwissenschaft ist die Leistungsfähigkeit der Selbstregulierung durch organisierte Wirtschaftsinteressen vor allem in nationalen sektoralen Zusammenhängen untersucht worden. Daneben haben auch internationale Studien gezeigt, dass Unternehmen auch an transnationalen Arrangements beteiligt sind
(7). Das Ziel hier ist nicht nur zu demonstrieren, dass privatwirtschaftliche Akteure willens und kompetent sind, bestimmte Formen internationaler Selbstregulierung zu errichten, sondern auch, die Bedingungen zu spezifizieren, unter denen diese Arrangements mehr oder weniger gut funktionieren. Im folgenden wird über ein Fallbeispiel eines solchen Arrangements berichtet.

\section{Das Beispiel Sicherheit beim Umgang mit Farbstoffen}

Ein interessantes Beispiel für Selbstregulierung in einem Klub multinationaler Unternehmen sind die Bemuihungen der Toxicological Association of Dyes and Organic Pigments Manufactures (ETAD), die 1974 errichtet wurde um Selbstverantwortung in der Farbstoffindustrie unter Beweis zu stellen. Selbstregulierung innerhalb der ETAD umfasst die Erzeugung von Informationen und die Formulierung und Überwachung von Verhaltensregeln. Die Mitgliedsfirmen verpflichten sich, das gemeinsame Ziel einer Minimierung der Gefahren im Umgang mit Farbstoffen anzustreben. Konkret bedeutet dies, dass von den Mitgliedsfirmen systematisch Daten über öko-toxikologische Eigenschaften von Farbstoffen gesammelt werden und mit Empfehlungen über den korrekten Umgang mit diesen Produkten verbreitet werden, um letztlich Risiken am Arbeitsplatz und in der Umwelt zu minimieren.

Daneben leistet die ETAD auch einen beträchtlichen Beitrag zur Produktkennzeichnung, die in der Regel staatlich reguliert ist. Ohne die Informationen über chemische Substanzen, die durch die Sicherheitsblätter mobilisiert werden, wäre es schwer, Produkte überhaupt auf effiziente und effektive Weise zu kennzeichnen. Hier assistiert die ETAD also bei staatlichen Politiken, die von intergouvernementalen Organisationen initiiert und durchgeführt werden. Ferner kontrolliert sie, ob diese Arrangements auch richtig funktionieren. Hierzu werden regelmäßig Tagungen organisiert. Wichtig dabei ist, dass die Verantwortung für die Tests letztlich bei den Firmen verbleibt und die ETAD nur Methoden evaluiert. Wenn nötig, führt sie jedoch auch investigative Studien durch. In der Zwischenzeit wurden diese Testrichtlinien auch von einschlägigen internationalen Organisationen anerkannt, insbesondere von der OECD.

Die allgemeinen Ziele der ETAD wurden in einem Ethik-Kodex festgelegt, der im Jahre 1989 von ihren Mitgliedsfirmen angenommen und im Jahre 1993 weiterentwickelt wurde. Obwohl die ETAD letztlich nicht in der Lage ist, den Kodex am Ende auch autoritativ durchzusetzen, ist ihr klar bewusst, dass ihre Glaubwürdigkeit davon abhängt, ob sie als Organisation das Verhalten ihrer Mitglieder bezüglich dieser Normen tatsächlich überwacht und auf Klagen und Einsprüchen reagiert.

\section{- Private Normen mit Reichweite}

Interessant ist, dass diese privaten Normen global gültig sind und somit auch für Exporte in Länder gelten, in denen noch keine nationalen Regulierungsstrukturen existieren. Hierbei kam es auch schon zu Sanktionen, und Mitgliedsfirmen, die sich nicht an den Kodex hielten, wurden aus der Vereinigung ausgeschlossen. Hieraus kann man schließen, daß die übrigen Mitgliedsfirmen die im Kodex vereinigten Umweltschutzgesichtspunkte letztlich doch - mehr oder weniger - ernst nehmen.

Die Kapazität zur Selbstregulierung verweist auf die Organisationsstruktur der ETAD. Die Vereinigung basiert auf Direktmitgliedschaften von gegenwärtig 38 führenden Firmen in der chemischen Industrie. Trotz globaler Orientierung konzentriert sich die nationale Herkunft der Mitgliedsfirmen im Wesentlichen auf sehr wenige Industrieländer. Resultat ist, dass die selbstdisziplinären Bemühungen und die hohen Standards in Ländern mit fortgeschrittenen Regulierungssystemen, in denen auch die wichtigsten Chemieproduzenten beheimatet sind, weitgehend effektiv umgesetzt werden. Dagegen besteht weiterhin das Risiko, dass limitierte Regulierungskapazitäten in der Dritten Welt von den Firmen ausgenutzt werden könnten, um eine Politik der doppelten Standards zu praktizieren. Dies stellt hohe Anforderungen an die Überwachungskapazität der ETAD, die letztlich durch die jeweiligen Mitgliedsfirmen unterstiutzt werden muss. Hierbei ist es von großer Hilfe, daß der Sektor relativ klein ist und dass die Wettbewerber mit ihren globalen Organisationsstrukturen meist in der Lage sind, die Aktivitäten ihrer Konkurrenten zu überschauen und über abwegiges Verhalten zu berichten.

\section{- Interne Überwachung}

Das Hauptelement der ETAD-Strategie ist, freiwillige Selbstregulierungsarrangements parallel zur Harmonisierung nationaler Regulierungssysteme anzubieten. Obwohl die Arrangements von Regierungen, zwischenstaatlichen Organisationen und internationalen Umweltgruppen überwacht werden, hat die Vereinigung absichtlich vermieden, bei 
der Überwachung des Verhaltens ihrer Mitgliedsfirmen auf die Unterstïtzung anderer interessierter Parteien zurückzugreifen. Dieses Arrangement hat den Vorteil, dass in diesem Regulierungskontext spezifische Informationen mobilisiert werden können, die außerhalb der Firmen nur schwer und vor allem nicht vollständig erfasst werden können. Auf der anderen Seite zeigte die ETAD auch wegen des Problems der Geheimhaltung firmeninterner Informationen wenig Kooperationsbereitschaft mit nichtindustriellen Organisationen. Die Industrie präferiert generell eigene unabhängige Mechanismen, ohne hierbei auf formellen Konsens mit relevanten nationalen, regionalen oder globalen Regierungsbehörden zu warten.

\section{- Kapazitäten und Kontingenzen}

Die Steuerung internationaler Zusammenhänge und die Lösung globaler Probleme wurde lange als Reservat zwischenstaatlicher Kooperation und intergouvernementaler Organisationen betrachtet. Dass Organisationen aus der Privatwirtschaft zur Bereitstellung von öffentlichen Steuerungs- und Regelungsleistungen beitragen und diese Kapazitäten auf globaler Ebene einsetzen, hat bisher nur wenig Aufmerksamkeit gefunden.

In einer Reihe von Organisationen, vom kleinen Klub multinationaler Firmen über größere Branchenverbände bis hin zur umfassenden Organisation der Weltunternehmerschaft, werden viele Varianten von Klubgütern produziert, deren Nutzen ganz deutlich auf die beteiligten Mitglieder ausgerichtet ist. Darüber hinaus enthalten viele dieser Regelwerke neben den Klubgutelementen auch eine öffentliche Komponente, indem mit ihrem Wirken positive Handlungsexternalitäten für die Weltgesellschaft verbunden sind. Regulierungsziele sind hierbei sowohl auf das reibungslose Operieren von Märkten als auch auf die Korrektur von Marktversagen gerichtet. Wichtig ist dabei, zwischen marktverbessernder und marktersetzender Steuerung und Regulierung zu unterscheiden.

Der dargestellte Fall zeigt, dass die positiven ,öffentlichen Effekte" dieser Handlungsstrategien in der Regel auch offizielle Ziele dieser privaten Organisationen und nicht einfach nichtintendierte Nebeneffekte darstellen. In diesem Kontext liegt der Basisanreiz meist darin, öffentliche Regulierung zu vermeiden, die strenger sein könnte als Selbstregulierung. In einigen Branchen besteht ein besonderer Anreiz darin, das relativ negative Image in der Öffentlichkeit zu verbessern. Unter diesen Bedingungen wird öffentliche Regulierung durch die Industrie als eine latente kollektive Bedrohung wahrgenommen, der langfristig nur durch eine effektive Selbstdisziplin bei umweltund gesundheitsschutzrelevanten Aktivitäten begegnet werden kann.

\section{Bedeutung öffentlich-institutio- neller Infrastrukturen}

Selbstregulative Arrangements sind jedoch sehr selten vollkommen selbsttragende Konstruktionen. In der Regel sind sie in ausgedehnte öffentlich-institutionelle Infrastrukturen eingebettet. Wirksames privates Handeln ist meist auf öffentliche Anerkennung angewiesen. Häufig werden private Organisationen auch durch staatliche Behörden zur Selbstregulierung ermuntert oder bekommen solche Aufgaben sogar offiziell delegiert. Bestimmte Regulierungsformen zeigen genau in diese Richtung, unter anderem deshalb weil die privaten Akteure oft über sehr spezialisierte Ressourcen wie Insiderwissen verfügen, die ihnen erlauben, effektivere Überwachungsmechanismen aufzubauen als dies von Regierungen geleistet werden könnte.

Unmittelbar einsichtig ist, dass eine wirksame Überwachung und Sanktionen eher in kleineren Organisationen umsetzbar sind, in denen die Mitglieder sich intensiver beobachten können. Doch selbst in kleinen freiwilligen Organisationen ist es nicht immer möglich, heroisch-hierarchisch zu sanktionieren. In diesem Falle können jedoch auch indirekte Maßnahmen angemessen sein, die meist horizontal durch Gruppendruck und sogenannte inkrementelle Sanktionen (8) wirken. Eine dieser subtilen Formen sozialer Kontrolle ist Reputationsverlust durch negative Publizität. Dies legt nahe, dass globale Normen in vielen Situationen auch indirekt und netzwerkartig über ein verteiltes System institutioneller Steuerungsarrangements durchgesetzt werden können.

Öffentliche Regulierung durch private Organisationen hängt demnach allgemein von deren Überwachungs- und Sanktionskapazität, gleichzeitig jedoch auch von einer funktionierenden komplementären Hintergrundkontrolle durch öffentliche Institutionen, Verbrauchergruppen und wissenschaftliche Gesellschaften ab. Insbesondere diese meist wenig sichtbare institutionelle Infrastruktur demokratisch-pluralistischer Gesellschaften wird leicht übersehen. Aber erst auf ihrer Basis kann das Damoklesschwert der negativen Publizität für potenzielle Regelabweichler eine Art letzte Kontrollinstanz darstellen.
Wenn also relevante Beiträge zur Lösung globaler Probleme bereits aus so problematischen Bereichen wie jenen des profitorientierten, rationalkalkulierenden freien Unternehmertums erwartet werden können, so kann man dies als Hinweis sehen, dass noch viele unentdeckte Potenziale für private Steuerung in manchen Politik- und Organisationsfeldern schlummern, die auf ihre Entdeckung warten. Weitere Forschung in diesem Bereich könnte uns ein realistischeres Bild über die vielfältigen Rollen liefern, die private Organisationen in der globalen Politik wahrnehmen, und das Thema der globalen Steuerung könnte somit in einen breiteren Kontext gestellt werden.

\section{Anmerkungen}

(1) Vgl. Marin, B./ Mayntz, R. (eds.): Policy Networks. Empirical Evidence and Theoretical Considerations. Frankfurt a. M. 1991. (2) Vgl. Streeck, W.: Interessenverbände als Hindernisse und Vollzugsträger öffentlicher Politik. In: Scharpf, F.W./ Brockmann, M. (Hrsg.): Institutionelle Bedingungen der Arbeitsmarkt- und Beschäftigungspolitik. Frankfurt/M. 1983. (3) Vgl. z.B. Rosenau, J.N./ Czempiel, E.-O. (eds.): Governance without Government: Order and Change in World Politics, Cambridge MA 1992; Young, O.R. (ed.): Global Governance. Drawing Insights from the Environmental Experience, Cambridge MA 1997; Charnowitz, S.: Two Centuries of Participation: NGOs and International Governance, in: Michigan Journal of International Law, Vol. 18 (1997), S. 183-286. (4) Vgl. Ruggie, J.G.: Collective Goods and Future International Collaboration, in: The American Political Science Review, Vol. 66 (1972), S. 874-893.

(5) Vgl. z.B. Olson, M.: The Logic of Collective Action: Public Goods and the Theory of Groups, Cambridge MA 1965; Schmitter, P.C./ Streeck, W.: The Organization of Business Interests. A Research Design to Study the Associative Action of Business in Advanced Industrial Societies of Western Europe, WZB discussion paper IIM/ LMP 81-13, Berlin 1981, S. 48-61.

(6) Vgl. dazu auch Fuchs, P.: Transnationale Konzerne als umweltpolitische Akteure. In: Ökologisches Wirtschaften Nr. 2/98, S. 25-27.

(7) Vgl. Cutler, C./ Haufler, V./ Porter, T. (eds.): Private Authority and International Affairs. New York 1999.

(8) Vgl. Coleman, J.S.: Foundations of Social Theory. Cambridge MA 1990.

\section{Die Autoren}

Dr. Volker Schneider ist Professor für Staatstheorie an der Universität Konstanz.

Kontakt: Tel. 07531/ 88-2320, Fax -4006, E-mail:Volker.Schneider@uni-konstanz.de Karsten Ronit, Ph.D., ist Associate Professor am Institute of Political Science an der Universität Kopenhagen. Kontakt: Tel. 0045-35-32-3402, Fax -3399, E-mail: kr@ifs.ku.dk 
(c) 20I0 Authors; licensee IÖW and oekom verlag. This is an article distributed under the terms of the Creative Commons Attribution Non-Commercial No Derivates License (http://creativecommons.org/licenses/by-nc-nd/3.o/), which permits unrestricted use, distribution, and reproduction in any medium, provided the original work is properly cited. 